\title{
Incubation Time, Cultivar, and Storage Duration Affect Onion Lachrymatory Factor Quantification
}

\author{
David E. Kopsell ${ }^{1}$ and William M. Randle ${ }^{2}$ \\ Department of Horticulture, The University of Georgia, Athens, \\ GA 60602-7273

\section{Norman E. Schmidt \\ Department of Chemistry, Georgia Southern University, Statesboro, GA 60460-8046}

Additional index words. Allium cepa, trans-(+)-S-(1-propenyl)-L-cysteine sulfoxide, $(Z, E)$ propanethial $S$-oxide, thiosulfinates, in vivo hydrolysis, gas chromatography, flavor

\begin{abstract}
The lachrymatory factor $[\mathrm{LF},(Z, E)$ propanethial $S$-oxide] is a direct product of 1-propenyl cysteine sulfoxide (1-PRENCSO) hydrolysis and dominates onion flavor when present in high concentrations. To evaluate $\mathbf{L F}$ as a potential means of assessing flavor quality, two onion cultivars were greenhouse-grown and the bulbs stored for 4 months at $3 \pm 1{ }^{\circ} \mathrm{C}, 70 \%$ relative humidity. Onions were evaluated at monthly storage intervals for LF development in bulb macerates following a 120 seconds incubation time. When LF was compared to amounts of 1-PRENCSO hydrolysis, we found that LF was severely underestimated. The relationship of $L F$ and 1-PRENCSO also varied between cultivars during storage. As 'Granex 33' was stored for longer periods, the amount of LF measured at 120 seconds more closely reflected the amount of 1-PRENCSO hydrolyzed. LF from 'Dehydrator \#3', however, was consistently underestimated regardless of storage time. Therefore, a second experiment was conducted using individual bulbs of two onion cultivars in an attempt to determine the optimal incubation time for $\mathrm{LF}$ quantification. Maximum LF among bulbs was generally detected 5-10 seconds after tissue maceration for 'Dehydrator' and after 15-30 seconds for 'Sweet Vidalia'. The amount of LF quantified between 5 and 120 seconds decreased linearly for nine of ten bulbs of 'Dehydrator', but this trend was less apparent for 'Sweet Vidalia'. A uniform LF incubation time for individual bulbs, therefore, may not be possible for all cultivars. These data show a complex relationship among and within onion cultivars for 1-PRENCSO hydrolysis and the formation of LF in onion macerates.
\end{abstract}

Onions (Allium cepa L.) are primarily consumed for their flavors. The characteristic onion flavor develops when the tissue is cut or damaged. The enzyme alliinase (EC 4.4.1.4), which is located in the vacuole, is released to hydrolyze the flavor precursors, collectively known as $S$-alk(en)yl-L-cysteine sulfoxides (ACSOs), located in the cytoplasm (Block, 1992; Lancaster and Collin, 1981). The three naturally occurring onion ACSOs are trans(+)-S-(1-propenyl)-L-cysteine sulfoxide (1PRENCSO), (+)-S-methyl-L-cysteine sulfoxide (MCSO), and (+)-S-propyl-L-cysteine sulfoxide (PCSO; Lancaster and Boland, 1990). Initial products of the hydrolytic reaction are sulfenic acids that then go on to produce the lachrymatory factor (LF), thiosulfinates, ammonia, and pyruvic acid.

The thiosulfinates are responsible for the different flavor attributes associated with raw onions when consumed (Block, 1986; Randle et al., 1994). The thiosulfinates rearrange over

Received for publication 27 Mar. 2001. Accepted for publication 2 Oct. 2001

${ }^{1}$ Former Graduate Assistant. Current address: Dept. of Plant Biology, Univ. of New Hampshire, Durham, NH 03824

${ }^{2}$ Professor. time and produce disulfides and other S-compounds. $(Z, E)$ Propanethial $S$-oxide, or the LF, arises from the hydrolysis of 1-propenyl cysteine sulfoxide (1-PRENCSO) and is responsible for the mouth burn and heat associated with onion consumption when in solution (Block, 1992; Randle et al., 1994). Sensory attributes from the LF can be overwhelming and dominate the experience when consuming onions with 1-PRENCSO in high concentration.

Many methods have been reported for LF attempts to separate the components of onion volatiles, including the LF, employed steam distillation and chromatography separations (Spare and Virtanen, 1963). These methods, however, were qualitative rather than quantitative. Saghir et al. (1963) developed a gas chromatography procedure using an internal standard to quantify mono- and disulfides in onion headspaces that later proved to have too long of a run time to capture the LF. Another LF quantification method used hexane extraction and spectrophotometric absorbance at 254 nm (Freeman and Whenham, 1975). However, because other compounds were also extracted in hexane and absorbed at $254 \mathrm{~nm}$, this method proved to be unreliable for LF quantiqualification and quantification. The earliest fication (Schmidt et al., 1996). Tewari and Bandyopadhyay (1975) developed a thin layer chromatography (TLC) procedure for LF quantification, but TLC is a slow, cumbersome process. Using TLC, LF was reported to be maximally produced within 2 min of tissue maceration, and to rapidly disappeared thereafter (Lukes, 1971). High-performance liquid chromatography (Si-HPLC) can separate many of the onion thiosulfinates, but does not quantify the LF because it co-eluted with the $\mathrm{C}_{6}$ thiosulfinates (Block, 1992; Block et al., 1992). Using gas chromatography-mass spectral separation, hot injection port and column temperatures caused onion chemicals to rearrange and created artifacts (Block et al., 1992). However, by using lower temperatures during GC injection and separation, LF was reliably detected but not quantified (Block et al., 1992).

Schmidt et al. (1996) investigated and developed a rapid GC method to optimize and quantify the LF by utilizing an internal standard. They reported maximum LF detection at their initial assessment time following a 2-min macerate incubation. Beyond $2 \mathrm{~min}$, LF decreased and they speculated this was due to volatilization, hydrolysis, or reduction (Schmidt et al., 1996). The most recently published technique for analyzing onion thiosulfinates and LF employed supercritical fluid extraction (Calvey et al., 1997). However, LF detection was low due to its volatility and inefficient trapping on glass beads at $0{ }^{\circ} \mathrm{C}$.

Because the method of Schmidt et al. (1996) appeared to be the most reliable and rapid procedure for quantifying LF, an experiment (1) was conducted using their method to assess LF changes that may occur before and during bulb storage using two onion cultivars. However, when the LF was compared to the amount of 1-PRENCSO hydrolysis in the macerates, an inherent problem with the time of incubation became apparent. A second experiment (2) was conducted to determine the time of maximum LF detection in onion macerates and its relationship to 1-PRENCSO hydrolysis.

\section{Materials and Methods}

Experiment 1. Two short-day onion cultivars, 'Dehydrator \#3' (Sunseeds, Hollister, Calif.) and 'Granex 33' (Asgrow, Kalamazoo, Mich.), were selected based on their previous reported flavor changes during storage (Kopsell and Randle, 1997). In Dec. 1997, each cultivar was seeded into Fafard 3-B substrate (Fafard, Anderson, S.C.), watered as needed, and fertilized with $20 \mathrm{~N}-20 \mathrm{P}-20 \mathrm{~K}$ nutrient solution (Grace-Sierra Co., Milpitas, Calif.) every 7 to $10 \mathrm{~d}$. Seedlings were greenhouse grown $\left(34^{\circ} \mathrm{N}\right.$ lat.) with temperatures of $28{ }^{\circ} \mathrm{C}$ day $/ 16{ }^{\circ} \mathrm{C}$ night for 7.5 weeks before transplanting into growing boxes containing Fafard 3-B. Sixteen plants of each cultivar were planted into about forty, $46 \times 46 \times 14-\mathrm{cm}$ boxes spaced $10 \mathrm{~cm}$ on center. About $100 \mathrm{~mL}$ of a full-strength Hoagland and Arnon (1950) solution was applied weekly to each plant until the bulbs were harvested. The plants were watered as needed throughout the experiment. 
Bulbs of each cultivar were harvested from 5-10 May 1998 when $>50 \%$ of the plants had foliar lodging. Bulb size and maturity were similar to field-grown onions. The plants were uprooted and laid in the boxes for several days. As the leaves senesced and browned, they and the roots were removed and the bulbs placed into mesh bags and hung in the greenhouse to dry for $7 \mathrm{~d}$. About 18 uniform bulbs from each cultivar were selected and placed into 16 mesh bags each.

Prior to storage, four, 10-bulb replicates of each cultivar were set aside for initial analysis. The remaining 18-bulb bags were placed into refrigerated storage $\left(3 \pm 1{ }^{\circ} \mathrm{C}, 70 \%\right.$ relative humidity) using a split-block design with four blocks. Blocks were the different quadrants of the cooler, cultivars were the main plots, and months of storage were the subplots. All cultivars were stored for 4 months. At monthly intervals, four bags of onions from each cultivar were removed from storage and the bulbs were allowed to acclimate to room temperature for $24 \mathrm{~h}$ prior to analysis. Ten intact, uniform bulbs from each cultivar were selected from each bag for 1-PRENCSO hydrolysis and LF quantification following bulb maceration.

Prior to analysis, each of the 10 bulbs was cut in half from top to bottom and the 10 halves combined into a single sample. The halves from one group were used to quantify the amount of intact 1-PRENCSO present prior to tissue maceration according to the method of Kopsell et al. (1999). The other half was used to determine the amount of 1-PRENCSO hydrolysis according to Lancaster et al. (1998) and the amount of LF produced after tissue maceration according to the method of Schmidt et al. (1996). Thin wedges of the 10 bulbs were juiced in a pneumatic press and a $0.5-\mathrm{mL}$ aliquot was taken after $120 \mathrm{~s}$ incubation and immediately placed into $10 \mathrm{~mL} 12$ methanol : 3 water $(\mathrm{v} / \mathrm{v})$ to stop the enzymatic reaction (Lancaster et al., 1998). To each $10.5-\mathrm{mL}$ methanol/juice sample, $S$-methyl glutathione $(0.5 \mathrm{mg}), \gamma$-L-glutamyl-L-glutamic acid $(0.2$ $\mathrm{mg}$ ), and ( \pm )-S-1-butyl-L-cysteine sulfoxide $(1.0 \mathrm{mg})$ were added as internal standards. Samples were then prepared for 1-PRENCSO quantification by HPLC. These data were then subtracted from the 1-PRENCSO amount found in the intact tissue and reported as hydrolyzed 1-PRENCSO.

Lachrymatory factor quantification was performed on 0.30-cm-thick wedges cut top to bottom from each 10-bulb sample and juiced in the pneumatic press (Schmidt et al., 1996). The juice was collected in a $400-\mathrm{mL}$ beaker, and a 5.0-mL aliquot was sampled $120 \mathrm{~s}$ after tissue maceration and immediately placed into a 20-mL test tube containing 4.0 mL of HPLC grade methylene chloride and $1.0 \mathrm{~mL}$ of $0.01 \%$ of the internal standard $p$-cymene in methylene chloride (v/v; Schmidt et al., 1996). The extract was centrifuged $(1381 \mathrm{~g})$ for $\approx 2 \mathrm{~min}$ and the lower organic layer was concentrated to $\approx 0.5 \mathrm{~mL}$ with a stream of nitrogen gas. The concentrated sample was then placed in an ice bath and a 1.0- $\mu \mathrm{L}$ sample was injected into a GC (Hewlett-Packard 5890 Series II, Palo
Alto, Calif.). Separation was achieved using a head pressure of $1.0 \mathrm{PSI}\left(1.2 \mathrm{~mL} \cdot \mathrm{min}^{-1}\right)$ with $99.999 \% \mathrm{He}$ on a $5 \mathrm{~m} \times 0.54-\mathrm{mm} \mathrm{OV}-1$ column. The oven temperature was $60^{\circ} \mathrm{C}$ for 1 min and then increased $5{ }^{\circ} \mathrm{C} / \min$ to $200{ }^{\circ} \mathrm{C}$. The injector temperature was maintained at 3 ${ }^{\circ} \mathrm{C}$ greater than the oven temperature. A flame ionization detector maintained at $250{ }^{\circ} \mathrm{C}$ was used. The LF response was integrated (HewlettPackard 3396 Series II Integrator) and peak assignment was carried out by comparing retention times with an authentic LF standard synthesized according to the method of Block et al. (1996). LF concentration was determined by comparing GC peak areas of the compound and the $p$-cymene internal standard for the same sample.

Experiment 2. Freshly harvested onion bulbs were obtained from industry sources ('Sweet Vidalia' from Terry Gerald, Statesboro, Ga., and 'Dehydrator' from Rogers Foods, Turlock, Calif.). 'Sweet Vidalia' is a mild, yellow Granex-type onion, while 'Dehydrator' is a high solids, pungent onion. Ten, single-bulb replications were selected for each cultivar. 1-Propenyl cysteine sulfoxide was determined as in Expt. 1 with the following exception. The amount of 1-PRENCSO hydrolyzed and LF produced in the onion macerates was determined after $5,10,15,30,60,90$, and $120 \mathrm{~s}$ of incubation to determine the time of maximum LF production.

LF was quantified using a modified procedure of Schmidt et al. (1996) to lessen the time required for $\mathrm{LF}$ analysis. A $1.0-\mathrm{mL}$ aliquot of juice was taken from the onion macerate following $5,10,15,30,60,90$, or $120 \mathrm{~s}$ incubation and immediately placed into a test tube containing $1.0 \mathrm{~mL}$ HPLC grade methylene chloride with $0.01 \% \mathrm{~m}$-xylene (v/ v) as an internal standard. $M$-xylene was found to be similar as an internal standard to the $p$-cymene used in Expt. 1, yet resolved earlier (data not shown). The test tubes were capped with a rubber stopper and repeatedly hand inverted for $10-15 \mathrm{~s}$. The test tube extracts were then centrifuged $(1381 \mathrm{~g}$ ) for $\approx 2$ min and cooled in an ice bath. One microliter of the lower organic layer was injected on the GC for separation. The GC used a split injector maintained at $200{ }^{\circ} \mathrm{C}$. Separation was achieved using a head pressure of $0.5 \mathrm{PSI}$ $\left(0.6 \mathrm{~mL} \cdot \mathrm{min}^{-1}\right)$ with $99.999 \% \mathrm{He}$ on a $5 \mathrm{~m} \times$ 0.54-mm OV-1 column. The column was maintained at an isothermal temperature of $60{ }^{\circ} \mathrm{C}$. Detection was achieved using flame ionization at $250{ }^{\circ} \mathrm{C}$. The $\mathrm{LF}$ response was integrated and peak assignment was carried out as in Expt. 1. LF concentration was determined by comparing GC peak areas of the compound and the $m$-xylene internal standard for the same sample. Run times were $\approx 2$ min per sample. Results obtained were similar to those of Schmidt et al. (1996) (data not shown).

Data were analyzed using the GLM and Fisher procedures of SAS (Cary, N.C.). Linear and polynomial regressions were performed on the LF and 1-PRENCSO data among months of storage and with onion macerate incubation time.

\section{Results and Discussion}

Experiment 1: Changes in LF during bulb storage. Data analyzed by GLM showed that the LF produced from macerated bulbs was found to differ between cultivars $(P=0.1)$, among months of bulb storage $(P=0.001)$, and for the interaction between cultivars and months of storage $(P=0.007)$. 1-Propenyl cysteine sulfoxide hydrolyzed in the onion macerates differed between cultivars $(P=$ $0.001)$ and among months of bulb storage $(P=$ $0.04)$. Onion flavor changes as measured by pyruvic acid content (Kopsell and Randle, 1997) and flavor precursor content (Kopsell et al., 1999) have been reported during bulb storage.

Prior to storage, the amounts of 1PRENCSO hydrolyzed were substantially more than the amounts of LF produced $120 \mathrm{~s}$ after tissue maceration (Table 1). For 'Granex 33', $11.48 \mu \mathrm{mol} \cdot \mathrm{mL}^{-1}$ of 1-PRENCSO juice were hydrolyzed. However, only 6.96 $\mu \mathrm{mol} \cdot \mathrm{mL}^{-1}$ of LF juice were captured, yielding a 1-PRENCSO : LF ratio of 1.7:1. The difference between LF produced and 1-PRENCSO hydrolyzed was even greater for 'Dehydrator \#3' with a 1-PRENCSO : LF ratio of 2.9:1. 'Dehydrator \#3' also hydrolyzed almost three times the 1-PRENCSO as did 'Granex 33', yet less than twice the LF was measured. These results suggested a problem in sampling for LF using a 120 -s macerate incubation time.

During storage, the amounts of 1PRENCSO hydrolyzed and LF detected changed as did their ratios. 'Granex 33' LF increased linearly $[\mathrm{LF}=6.10+0.91$ Month; $P$ $=0.001]$ while 'Dehydrator \#3' LF decreased, then increased following a quadratic trend [LF $=11.84-2.17$ Month + 0.34 Month ${ }^{2} ; P=$ 0.058 ] over 4 months (Table 1 ). Changes in LF behavior during storage have not been previously reported. Following each month of storage, the amounts of 1-PRENCSO hydrolyzed exceeded the amount of LF captured for each cultivar. By the second month of storage, however, the amount of LF captured $120 \mathrm{~s}$ after tissue maceration almost reflected the amount of 1-PRENCSO hydrolyzed (1.1:1) with 'Granex 33'. The 1.1:1 ratio then remained the same through 4 months of storage (Table 1). The amount of LF captured from 'Dehydrator \#3', however, was always less than half the amount of 1-PRENCSO hydrolyzed, regardless of the storage period.

To investigate this further, a preliminary experiment with a few remaining bulbs was performed where 1-PRENCSO and LF were sampled at earlier incubation times. 1PRENCSO hydrolysis was slower and less complete in 'Granex 33' when compared to 'Dehydrator \#3'. Differences in the amount of LF captured may be explained by the differences in the way 1-PRENCSO is hydrolyzed by each cultivar. After a 10 -s macerate incubation, only $64 \%$ to $70 \%$ of the 1-PRENCSO was hydrolyzed for 'Granex 33' while 93\% to 97\% was hydrolyzed for 'Dehydrator \#3'. Waiting $80 \mathrm{~s}, 70 \%$ to $90 \%$ of 1-PRENCSO was hydrolyzed in the 'Granex 33' macerates while continued hydrolysis in the "Dehydrator 
\#3' macerate was negligible. Because LF is a transient compound due to volatilization or reaction with other sulfenic acid by-products (Block, 1992), incubation time in the macerate can apparently affect its quantification based on the hydrolysis pattern. Block (1992) has shown that when 1-PRENCSO is hydrolyzed by alliinase, 1-propenyl sulfenic acid is formed and undergoes an immediate rearrangement to yield the LF. Although other thiosulfinates and zwiebelanes can be formed following this reaction, the vast majority of 1-PRENCSO products were detected as LF (Randle et al., 1994). Therefore, a close relationship between the amount of 1-PRENCSO hydrolyzed and LF produced should depend on the rate of 1PRENCSO hydrolysis and the incubation time for sampling. This can be seen in the ratios of 1-PRENCSO : LF for 'Granex 33' and 'Dehydrator \#3' following a 120 -s incubation. Because $95 \%$ of 1-PRENCSO was hydrolyzed within $5 \mathrm{~s}$, sufficient time existed for the LF to be lost to volatilization or degradation. LF is, therefore, underestimated at $120 \mathrm{~s}$ (Table 1). With 'Granex 33', 1-PRENCSO hydrolysis was slower and may account for a closer relationship with the LF detected at 120 s. Our preliminary data suggest that LF should be sampled before $120 \mathrm{~s}$ in order to prevent loss and better reflect the quantity of 1-PRENCSO hydrolyzed.

Experiment 2: Optimum incubation time for $L F$. Data analyzed by GLM showed that the level of LF detected and 1-PRENCSO hydrolyzed differed between the cultivars $(P$ $=0.001)$, among incubation times $(P=0.001)$, and for the interaction between cultivars and incubation times $(P=0.002)$. For most 'Dehydrator' bulbs, 1-PRENCSO was hydrolyzed mainly within $5 \mathrm{~s}$ of tissue maceration, although bulbs 2 through 4 had significant hydrolysis at longer incubation times (Table 3 ). The $\mu$ mols of maximum LF detection did not equate to and were often substantially lower than the amount of 1-PRENCSO hydrolyzed. LF measured also decreased linearly as incubation time increased for nine of 10 bulbs from 'Dehydrator' (Table 2). The systematic loss of LF from the macerate could be expected if 1PRENCSO is quickly and completely hydrolyzed due to degradation or volatilization. The LF maximum was detected for $90 \%$ of the bulbs when 'Dehydrator' was determined between 5-10 s after maceration. Fisher's exact test identified homogeneity $(P=0.01)$ in the LF maximum only between $10-15 \mathrm{~s}$ after tissue maceration. On average, only $41 \%$ of the maximum LF produced was detected at $120 \mathrm{~s}$ and this suggests that a 2-min incubation according to Schmidt et al. (1994) severely underestimated the LF maximum for this cultivar.

The time of maximum LF detection was more variable among the bulbs tested for 'Sweet Vidalia', and homogeneity was not found among any of the incubation times using Fisher's exact test. Three of the bulbs decreased linearly for LF, three bulbs increased, then decreased quadratically, and four exhibited no significant trend (Table 2). These trends could be expected if the maximum amount of

Table 1. Amount of $1-\mathrm{PRENCSO}^{\mathrm{z}}\left(\mu \mathrm{mol} \cdot \mathrm{mL}^{-1}\right.$ onion macerate juice) and lachrymatory factor (LF; $\mu \mathrm{mol} \cdot \mathrm{mL}^{-1}$ onion macerate juice) produced $120 \mathrm{~s}$ after tissue maceration and the ratio of 1-PRENCSO:LF for 'Granex 33' and 'Dehydrator \#3' during 4 months of storage at $3{ }^{\circ} \mathrm{C}, 70 \%$ relative humidity.

\begin{tabular}{|c|c|c|c|}
\hline Month & $\begin{array}{c}\mu \mathrm{mol} \text { of } \\
\text { 1-PRENCSO }\end{array}$ & $\begin{array}{c}\mu \mathrm{mol} \text { of } \\
\mathrm{LF}^{\mathrm{x}}\end{array}$ & $\begin{array}{c}\text { Ratio of } \\
\text { 1-PRENCSO : LF }\end{array}$ \\
\hline \multicolumn{4}{|c|}{ Granex 33} \\
\hline 0 & $11.48 \pm 2.68$ & $6.96 \pm 1.28$ & $1.7: 1$ \\
\hline 1 & $11.02 \pm 4.07$ & $7.44 \pm 2.54$ & $1.5: 1$ \\
\hline 2 & $10.23 \pm 3.78$ & $9.79 \pm 0.74$ & $1.1: 1$ \\
\hline 3 & $10.45 \pm 1.31$ & $9.59 \pm 0.60$ & $1.1: 1$ \\
\hline 4 & $11.93 \pm 3.49$ & $10.45 \pm 0.41$ & $1.1: 1$ \\
\hline \multicolumn{4}{|c|}{ Regression significance } \\
\hline Linear & NS & $P=0.001$ & \\
\hline \multicolumn{4}{|c|}{ Dehydrator \#3 } \\
\hline 0 & $30.11 \pm 3.16$ & $10.25 \pm 1.54$ & $2.9: 1$ \\
\hline 1 & $19.20 \pm 1.24$ & $8.28 \pm 0.91$ & $2.3: 1$ \\
\hline 2 & $22.50 \pm 2.55$ & $8.75 \pm 0.49$ & $2.6: 1$ \\
\hline 3 & $19.09 \pm 1.08$ & $8.78 \pm 0.42$ & $2.2: 1$ \\
\hline 4 & $25.79 \pm 0.97$ & $9.43 \pm 0.76$ & $2.7: 1$ \\
\hline \multicolumn{4}{|c|}{ Regression significance } \\
\hline Quadratic & NS & $P=0.058$ & \\
\hline
\end{tabular}

${ }^{2} 1$-PRENCSO $=$ trans-(+)-S-(1-propenyl)-L-cysteine sulfoxide.

${ }^{y}$ Average $\mu \mathrm{mol} \cdot \mathrm{mL}^{-1}$ onion macerate juice of four replications.

${ }^{\mathrm{x}}$ Average of duplicate injections.

${ }^{\mathrm{N}}$ Nonsignificant.

Table 2. Noncumulative amounts of lachrymatory factor $\left(\mathrm{LF} ; \mu \mathrm{mol} \cdot \mathrm{mL}^{-1}\right.$ onion macerate juice) measured at each hydrolysis incubation time of the individual onion bulbs of field-grown 'Sweet Vidalia' and 'Dehydrator'.

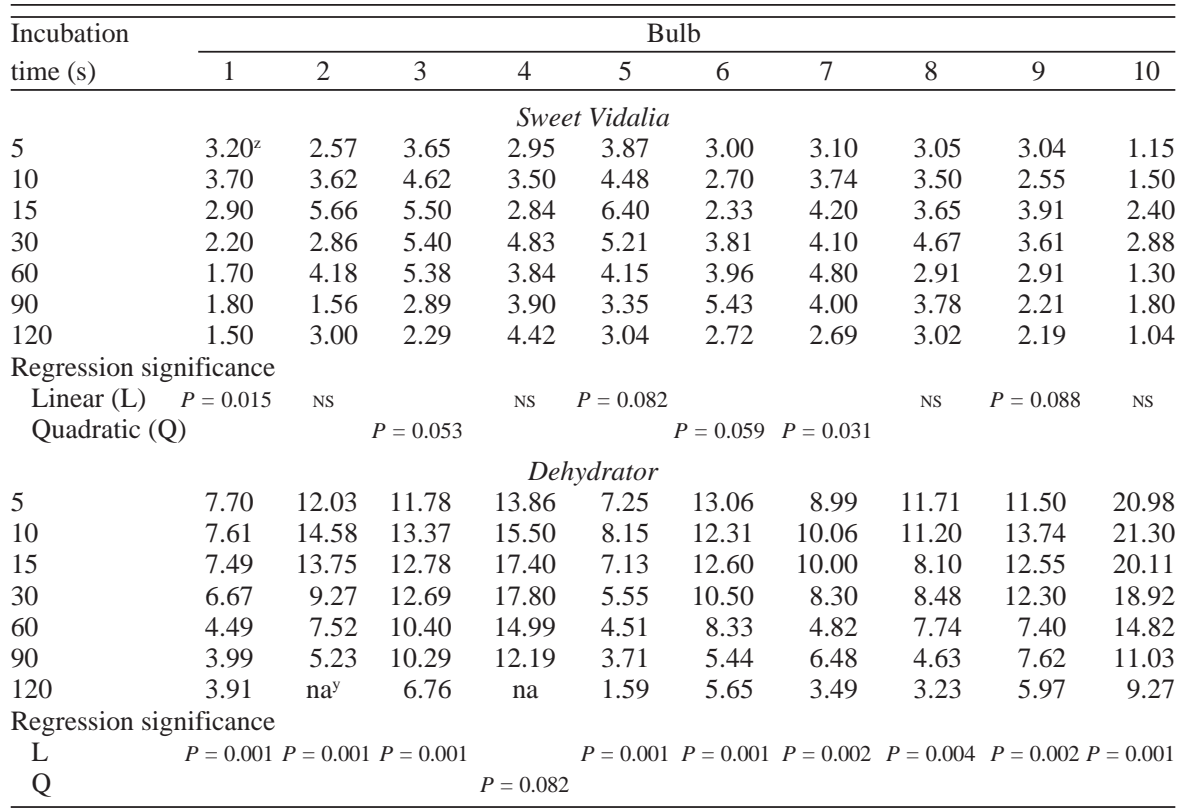

${ }^{\mathrm{z}}$ Average of duplicate sample injections.

y Not available.

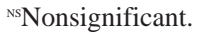

1-PRENCSO hydrolysis occurred later and at different times in the onion macerates (Table 3 ). The longer it takes to reach maximum 1PRENCSO hydrolysis, the more likely LF production and degradation will occur simultaneously. This would cause an underestimation of LF. Although time of maximum hydrolysis was variable for 'Sweet Vidalia', 70\% of the bulbs had maximum LF levels after 15$30 \mathrm{~s}$. On average, only $50 \%$ of the maximum LF produced was detected at $120 \mathrm{~s}$. Lukes (1971) measured a 50\% reduction in LF from 2 to $5 \mathrm{~min}$ and an $80 \%$ reduction in LF $15 \mathrm{~min}$ after tissue maceration using TLC. Allowing a 5-10-min incubation, Calvey et al. (1997) reported low LF quantification following supercritical fluid extraction.

Because LF is a direct reaction product and dominates onion flavor, its rapid quantification can be extremely valuable to researchers and marketers assessing onion flavor. Using an incubation time of $120 \mathrm{~s}$ after tissue maceration, LF could not be reliably quantified and was underestimated for two onion cultivars. Differences between and within the cultivars for 1-PRENCSO hydrolyzed and LF detected indicated that a shorter incubation period of the macerate was needed for maximum LF quantification. When macerates were analyzed as early as $5 \mathrm{~s}$ after juicing, LF 
Table 3. Noncumulative amounts of 1-propenyl cysteine sulfoxide (1-PRENCSO; $\mu \mathrm{mol} \cdot \mathrm{mL}^{-1}$ onion macerate juice) hydrolysis measured at each incubation time of the individual onion bulbs of field-grown 'Sweet Vidalia' and 'Dehydrator'.

\begin{tabular}{|c|c|c|c|c|c|c|c|c|c|c|}
\hline \multirow{2}{*}{$\begin{array}{l}\text { Incubation } \\
\text { time (s) }\end{array}$} & \multicolumn{10}{|c|}{ Bulb } \\
\hline & 1 & 2 & 3 & 4 & 5 & 6 & 7 & 8 & 9 & 10 \\
\hline \multicolumn{11}{|c|}{ Sweet Vidalia } \\
\hline 5 & 3.63 & 4.21 & 3.80 & 4.32 & 4.64 & 3.97 & 2.24 & 4.49 & 3.66 & 3.38 \\
\hline 10 & 4.59 & 4.78 & 4.03 & $\mathrm{na}^{\mathrm{z}}$ & 4.91 & 4.37 & 2.71 & 5.00 & 4.14 & na \\
\hline 15 & 4.73 & 5.28 & 4.32 & 4.74 & 5.15 & 4.62 & 3.02 & 5.43 & 4.51 & 3.45 \\
\hline 30 & 4.93 & 5.62 & 5.03 & 5.13 & 5.94 & 5.21 & 3.48 & 6.20 & 4.79 & 3.64 \\
\hline 60 & 4.93 & 5.74 & 5.72 & 5.43 & 6.71 & 6.13 & 4.12 & 6.30 & 5.01 & 4.00 \\
\hline 90 & 4.93 & 5.74 & 5.96 & 5.43 & 6.95 & 6.40 & 4.35 & 6.38 & 5.01 & 4.24 \\
\hline 120 & 4.93 & 5.74 & 6.12 & 5.47 & 7.03 & 6.68 & 4.40 & 6.38 & 5.01 & 4.24 \\
\hline \multicolumn{11}{|c|}{ Regression significance } \\
\hline Linear (L) & & $P=0.081$ & $P=0.004$ & $P=0.045$ & $P=0.005$ & $P=0.002$ & $P=0.007$ & $P=0.048$ & $P=0.054$ & $P=0.004$ \\
\hline \multicolumn{11}{|c|}{ Quad. (Q) $P=0.002$} \\
\hline \multicolumn{11}{|c|}{ Dehydrator } \\
\hline 5 & 13.04 & 14.20 & 13.16 & 12.90 & 12.64 & 13.60 & 15.09 & 14.62 & 15.90 & 13.96 \\
\hline 10 & 13.08 & 14.74 & 13.95 & na & 12.65 & 13.61 & 15.14 & 14.62 & 16.02 & 14.22 \\
\hline 15 & 13.12 & 16.06 & 14.01 & 13.49 & 12.75 & 13.62 & 15.14 & 14.63 & 16.14 & 14.24 \\
\hline 30 & 13.12 & 16.48 & 14.23 & 15.87 & 12.75 & 13.63 & 15.15 & 14.63 & 16.14 & 14.26 \\
\hline 60 & 13.13 & 16.48 & 14.23 & 16.42 & 12.75 & 13.63 & 15.17 & 14.64 & 16.22 & 14.26 \\
\hline 90 & 13.13 & 16.48 & 14.23 & 16.42 & 12.75 & 13.63 & 15.17 & 14.64 & 16.22 & 14.26 \\
\hline 120 & 13.13 & 16.48 & 14.23 & 16.47 & 12.75 & 13.63 & 15.17 & 14.64 & 16.22 & 14.26 \\
\hline \multicolumn{11}{|c|}{ Regression significance } \\
\hline $\mathrm{L}$ & NS & NS & NS & $P=0.068$ & NS & $P=0.096$ & $P=0.062$ & & $P=0.060$ & NS \\
\hline Q & & & & & & & & $P=0.010$ & & \\
\hline
\end{tabular}

${ }^{ }$Not available.

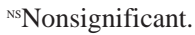

detection was variable between and within cultivars. Maximum LF was detected after 5$10 \mathrm{~s}$ for 'Dehydrator \#3' and appeared to be a suitable incubation period to sample this cultivar. 'Sweet Vidalia' was more variable for the incubation time required for maximum LF detection, although $70 \%$ of the bulbs tested had an LF maximum after 15-30 s. A uniform incubation time for LF quantification of individual bulbs may not be possible for all cultivars. Because the LF is an important attribute of onion flavor, individuals conducting breeding programs that emphasize flavor improvement or modification may want to utilize LF as a selection criterion. Its value as a selection tool should take into account an acceptable variance in optimum LF incubation times for each population of bulbs. However, using multiple bulbs per treatment sample may minimize bulb-to-bulb variation, making LF sufficiently accurate to assess the flavor quality of onions destined for consumption. This approach has been used to assess gross flavor intensity by measuring enzymatically formed pyruvic acid from multiple-bulb samples in test plots and for field sampling (Randle, 1992; Randle et al., 1998).

\section{Literature Cited}

Block, E. 1986. The chemistry of garlic and onions. Sci. Amer. 252:114-119.

Block, E. 1992. The organosulfur chemistry of the genus Allium-Implications for the organic chemistry of sulfur. Angew. Chem. Int. Ed. Engl. 31:1135-1178. chemistry: GC-MS analysis of thiosulfinates and related compounds from onion, leek, scallion, shallot, chive, and Chinese chive. J. Agr. Food Chem. 40:2431-2438.

Block, E., J.Z. Gillies, C.W. Gillies, A.A. Bazzi, D. Putman, L.K. Revelle, D. Wang, and X. Zhang. 1996. Allium chemistry: Microwave spectroscopic identification, mechanism of formation, synthesis, and reactions of $(E, Z)$-propanethial $S$ oxide, the lachrymatory factor of the onion. J. Amer. Chem. Soc. 118:7492-7501. DeOrazio, D. Sha, and E. Block. 1997. Allium chemistry: Supercritical fluid extraction and LCAPCI-MS of thiosulfinates and related compounds from homogenates of garlic, onion, and ramp. Identification in garlic and ramp and synthesis of 1-propanesulfinothioic acid $S$-allyl ester. J. Agr. Food Chem. 45:4406-4413.

Freeman, G.G. and R.J. Whenham. 1975. A rapid spectrophotometric method of determination of thiopropanal $S$-oxide (lachrymatory factor) in onions (Allium cepa L.) and its significance in
Block, E., D. Putman, and S.-H. Zhao. 1992. Allium

Calvey, E.M., J.E. Matusik, K.D. White, R flavour studies. J. Sci. Food Agr. 25:15291543.

Hoagland, D.R. and D.I. Arnon. 1950. The water culture method for growing plants without soil. California Agr. Expt. Sta. Circ. 347.

Kopsell, D.E. and W.M. Randle. 1997. Onion cultivars differ in pungency and bulb quality changes during storage. HortScience 32:1260-1263.

Kopsell, D.E., W.M. Randle, and M.A. Eiteman. 1999. Changes in the $S$-alk(en)yl cysteine sulfoxides and their biosynthetic intermediates during onion storage. J. Amer. Soc. Hort. Sci. 124:177-183.

Lancaster, J.E. and M.J. Boland. 1990. Flavor biochemistry, p. 33-72. In: H.D. Rabinowitch and J.L. Brewster (eds.). Vol. 3: Onions and allied crops. CRC Press, Boca Raton, Fla.

Lancaster, J.E. and H.A. Collin. 1981. Presence of alliinase in isolated vacuoles and of alkyl cysteine sulphoxides in the cytoplasm of bulbs of onion (Allium cepa). Plant Sci. Letters 22:169176.

Lancaster, J.E., M.L. Shaw, and W.M. Randle. 1998. Differential hydrolysis of alk(en)yl cysteine sulphoxides by alliinase in onion macerates: Flavour implications. J. Sci. Food Agr. 78:367-372.

Lukes, T.M. 1971. Thin-layer chromatography of cysteine derivatives of onion flavor compounds and the lachrymatory factor. J. Food Sci. 36:662664.

Randle, W.M. 1992. Sampling procedure to estimate flavor potential in onion. HortScience 27:1116-1117.

Randle, W.M., E. Block, M.H.Littlejohn, D. Putman, and M.L. Bussard. 1994. Onion (Allium cepa L.) thiosulfinates respond to increasing sulfur fertility. J. Agr. Food Chem. 42:2085-2088.

Randle, W.M., D.A. Kopsell, D.E. Kopsell, R.L. Snyder, and R. Torrance. 1998. Field sampling short-day onion for bulb pungency. HortTechnology 8:329-332.

Saghir, A.R., L.K. Mann, R.A. Bernhard, and J.V. Jacobsen. 1963. Determination of aliphatic mono- and disulfides and their distribution in the common food species. J. Amer. Soc. Hort. Sci. 84:386-398.

Schmidt, N.E., L.M. Santiago, H.D. Eason, K.A. Dafford, C.A. Grooms, T.E. Link, D.T. Manning, S.D. Cooper, R.C. Keith, W.O. Chance, M.D. Walla, and W.E. Cotham. 1996. Rapid extraction method of quantitating the lachrymatory factor of onion using gas chromatography. J. Agr. Food Chem. 44:2690-2693.

Spåre, C.G. and A.I. Virtanen. 1963. On the lachrymatory factor in onion (Allium cepa) vapours and its precursor. Acta Chem. Scand. 17:641650.

Tewari, G.M. and C. Bandyopadhyay. 1975. Quantitative evaluation of lachrymatory factor in onion by thin layer chromatography. J. Agr. Food Chem. 23:647-649. 